\title{
Searches for direct pair production of third generation squarks with the ATLAS detector
}

\author{
Priscilla Pani* on behalf of the ATLAS Collaboration \\ Nikhef, Amsterdam \\ E-mail: ppani@nikhef.nl
}

\begin{abstract}
Naturalness arguments for weak-scale supersymmetry favour supersymmetric partners of the third generation quarks with masses not too far from those of their Standard Model counterparts. Top or bottom squarks with masses less than a few hundred $\mathrm{GeV}$ can also give rise to direct pair production rates at the LHC that can be observed in the data sample recorded by the ATLAS detector. The talk presents recent ATLAS results from searches for direct stop and sbottom pair production based on $20.7 \mathrm{fb}^{-1}$ of integrated luminosity with the LHC operating at a pp centre-ofmass energy of $8 \mathrm{TeV}$.
\end{abstract}

XXI International Workshop on Deep-Inelastic Scattering and Related Subject -DIS2013, 22-26 April 2013

Marseilles, France

\footnotetext{
* Speaker.
} 


\section{Introduction}

Supersymmetry (SUSY) is a theory that provides an extension of the Standard Model. In this theory, a full set of new particles, supersymmetric partners of the known fermions and boson is introduced. The existence of the supersymmetric partner of the top quark (top squark or stop) is the main ingredient if SUSY has to provide a solution to the gauge hierarchy problem, since the stop is needed to cancel the loop diagrams involving top quarks, which are the dominant contribution to the divergence of the Higgs boson mass, provided that the $\tilde{t}_{1}$ mass is below the $\mathrm{TeV}$ range. These naturalness motivations and the wide range of experimental results obtained in the last years by the LHC experiments on SUSY searches favour a spectrum of SUSY particles that involve relatively light $\tilde{t}_{1}$ and $\tilde{b}_{1}$ and heavy $\tilde{u}, \tilde{d}, \tilde{s}, \tilde{c}$.

For this reason and especially after the discovery of a Higgs particle, searches for $3^{\text {rd }}$ generation squarks have have received increased attention in the HEP community. The most recent results from searches for scalar tops and bottoms direct production are summarised in this article. All analyses are based on $20.7 \mathrm{fb}^{-1}$ of integrated luminosity with the LHC operating at a pp centre-of-mass energy of $8 \mathrm{TeV}$. The data have been recorded with the ATLAS detector, a general-purpose experiment that detects and records collisions produced at the Large Hadron Collider (LHC) and has been extensively described in Ref. [1]. Requirements that ensure the quality of beam conditions, detector performance, and data are imposed.

The optimisation of these searches and the interpretation of the results have been all performed under few common assumptions. A R-parity conserving minimal supersymmetric extension of the SM is assumed, where all particles are produced in pairs and decay in a chain of SUSY and SM particles. The lightest SUSY particle (LSP) is stable and escapes the detector without interacting. In addition, the interpretation of the results is performed in terms of simplified models, where particles' unknown masses are free parameters and each decay is assumed with $100 \%$ Branching Ration (BR).

\section{Final states and general analysis strategy}

The $3^{\text {rd }}$ generation squarks are assumed to decay into the following final states.

$$
\begin{array}{ll}
\tilde{b}_{1} \rightarrow b \tilde{\chi}_{1}^{0} & \tilde{t}_{1} \rightarrow t \tilde{\chi}_{1}^{0} \\
\tilde{b}_{1} \rightarrow t \tilde{\chi}_{1}^{ \pm} \rightarrow t W^{ \pm} \tilde{\chi}_{1}^{0} & \tilde{t}_{1} \rightarrow b \tilde{\chi}_{1}^{ \pm} \rightarrow b W^{ \pm} \tilde{\chi}_{1}^{0}
\end{array}
$$

Many parameters determine the kinematics of the final state, as for example $m_{\tilde{t}_{1}}, m_{\tilde{b}_{1}}, \Delta m\left(\tilde{\chi}_{1}^{ \pm}, \tilde{\chi}_{1}^{0}\right)$, $\Delta m\left(\tilde{t}_{1} / \tilde{b}_{1}, \tilde{\chi}_{1}^{ \pm} / \tilde{\chi}_{1}^{0}\right)$ and the $\tilde{t}_{1}$ chirality. The presence of a $\tilde{\chi}_{1}^{0}$ is selected requiring $E_{\mathrm{T}}^{\text {miss }}$ in the event and final states with no, 1 or 2 are explored in different analyses in order to gain access to different ranges of such parameters.

All ATLAS SUSY searches make use of a common strategy that can be summarised in the following steps:

- Definition of a set of Signal enhanced Regions (SR), based on Montecarlo (MC) simulation only. Experimental data are kept blinded until the analysis is fully defined and frozen. 
- Definition of a set of Control Regions (CR), one for each major background of the analysis. These regions are designed to be as close as possible to the SRs, but orthogonal to them. They are mainly populated by a single background process and have low signal contamination. They are used to estimate the MC normalisation through a semi data-driven procedure.

- Estimation of the $\mathrm{CR} \rightarrow \mathrm{SR}$ Transfer Factors (TF) in order to extrapolate the data-driven MC normalisation to the SRs and validate them in the Validation Region (VR).

- Unblinding of the analysis and standard statistic procedure to determine whether an excess is observed. Otherwise, the results are interpreted in terms of limits on selected models through CLs calculation.

\section{Summary of sbottom searches}

The most recent results in direct sbottom pair production searches are extensively described in Refs. [2] and [3]. In particular, the latter analysis is a very powerful cut-and-count search based on $2 b$-jets $+E_{\mathrm{T}}^{\text {miss }}$ signature. Two sets of signal regions are defined to provide sensitivity to the kinematic topologies associated to different mass-splittings between the sbottom and the neutralino masses. In particular a set of the signal regions is defined increasing the tightness of a requirement on the con-transverse mass [4], that has proven to be a powerful variable for these searches and is defined in Eq. 3.1

$$
m_{C T}^{2}\left(v_{1} v_{2}\right)=\left[E_{T}\left(v_{1}\right)+E_{T}\left(v_{2}\right)\right]^{2}-\left[\overrightarrow{p_{T}}\left(v_{1}\right)-\vec{p}_{T}\left(v_{2}\right)\right]^{2}
$$

where $v_{1}, v_{2}$ are the b-jets in the event. An example of a SR distribution and the final exclusion results for this analysis are shown in Figure 1. Such a powerful signature has been used to set limits also in terms of $\tilde{t} \rightarrow b \tilde{\chi}_{1}^{ \pm}$searches, especially in the case of small mass difference between the $\tilde{\chi}_{1}^{ \pm}$and the $\tilde{\chi}_{1}^{0}$.

\section{Summary of stop searches}

A number of analyses have addressed stop pair production searches, on both $\tilde{t}_{1} \rightarrow t \tilde{\chi}^{0}$ and $\tilde{t}_{1} \rightarrow b \tilde{\chi}^{ \pm}$decay modes. In particular searches for the former are described in [5] and [6] where final states with no and 1 lepton are investigated, respectively. The summary of the interpretations of these searches, in terms of exclusion limits on simplified models, are shown in the right frame of Figure 3. Worth mentioning is the usage of a shape-fit strategy in the 1 lepton analysis in order to improve the sensitivity toward the diagonal region, i.e. $m_{\tilde{t}_{1}} \sim m_{\tilde{\chi}_{1}^{0}}$. In this case a bidimensional fit in the $\left(m_{T}, E_{\mathrm{T}}^{\text {miss }}\right)$ plane has been performed in order to maximise the signal over background discrimination power of these two variables and their shapes. In addition, the sensitivity of the analyses on the $\tilde{t}_{1}$ chirality have been studied and the results have been found to be little or not affected by different left-right mixtures.

Searches for the second major stop decay mode $\left(\tilde{t}_{1} \rightarrow b \tilde{\chi}^{ \pm}\right)$are extensively described in Refs.[7], [6] and [8] for no, 1 and 2 leptons final states respectively. Even under the simplified models assumptions, this decay mode is characterised by a 3-dimensional phase space determined 


\begin{tabular}{lll}
\hline Assumption & Motivation & Analyses \\
\hline$m\left(\tilde{\chi}^{ \pm}\right)=2 m\left(\tilde{\chi}^{0}\right)$ & gaugino universality & $1 / 2$ lep [6], [8] \\
$m\left(\tilde{t}_{1}\right)-m\left(\tilde{\chi}^{ \pm}\right)=10 \mathrm{GeV}$ & mass degeneracy & 2 lep [8] \\
$m\left(\tilde{\chi}^{ \pm}\right)-m\left(\tilde{\chi}^{0}\right)=5 / 10 / 20 \mathrm{GeV}$ & natural scenario (higgsino-like $\left.\tilde{\chi}_{1}^{ \pm}, \tilde{\chi}_{1}^{0}\right)$ & 0 lep $\left(2 \mathrm{~b}+E_{\mathrm{T}}^{\text {miss }}\right)[3]$ \\
$m\left(\tilde{\chi}^{ \pm}\right)=150 \mathrm{GeV}$ & fixed chargino mass (above LEP limit) & 1 lep [6] \\
$m\left(\tilde{\chi}^{0}\right) \sim 0$ & massless neutralino & 2 lep [8] \\
\hline
\end{tabular}

Table 1: Schematic description of the models investigated by ATLAS for the $\tilde{t}_{1} \rightarrow b \tilde{\chi}^{ \pm}$decay mode. In the last column the analysis with the major influence in a certain masses assumption is indicated.

by the masses of the three particles involved in the chain $\left(\tilde{t}_{1}, \tilde{\chi}_{1}^{ \pm}, \tilde{\chi}_{1}^{0}\right)$. ATLAS has pursued the strategy of cutting this space in bidimensional slices, according to certain physics-driven motivations. The wide multiplicity of final states addressed by the afore mentioned analyses allow a complementary coverage of these selected models, as summarised in Table 1

The 2 lepton analysis based its strategy on a very powerful distribution called the stransverse mass [9]:

$$
m_{T 2} \equiv \min _{\vec{q}_{T}+\vec{r}_{T}=\vec{p}_{T}^{\text {miss }}}\left\{\max \left[m_{T}\left(\vec{p}_{a}, \vec{q}_{T}\right), m_{T}\left(\vec{p}_{b}, \vec{r}_{T}\right)\right]\right\}
$$

where $m_{T}$ indicates the transverse mass, $a, b$ the two leptons in the event and $\vec{p}_{T}^{\text {miss }}$ the transverse missing momentum. The variable is calculated by minimisation over all the possible decompositions of $p_{T}^{\text {miss }}$ and presents a sharp kinematic endpoint, for $t \bar{t}$ events, at the $\mathrm{W}$ boson mass. $m_{T 2}$ is used as the final discriminating variable after enhancing the signal over background ratio with a requirement on the $\Delta \Phi_{b}$ between the $\mathbf{p}_{b}^{\ell \ell}$ and the $\vec{p}_{T}^{\text {miss }}\left(\Delta \phi_{b}<1.5\right)$, where the former represents the boost of the $W \mathrm{~s}$ or the $\chi \mathrm{s}$ system (Eq. 4.2) and the latter the missing transverse momentum in the

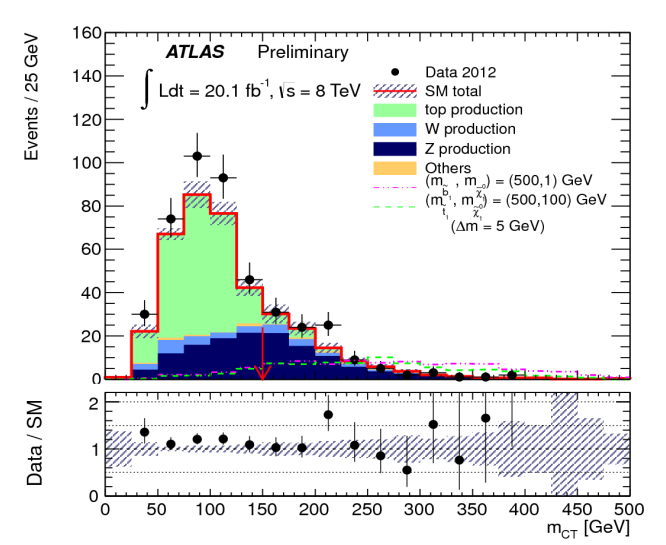

(a) $m_{C T}$ distribution in SRA with all the selections cuts applied except the $m_{C T}$ thresholds. The shaded band includes statistical and systematic uncertainties.

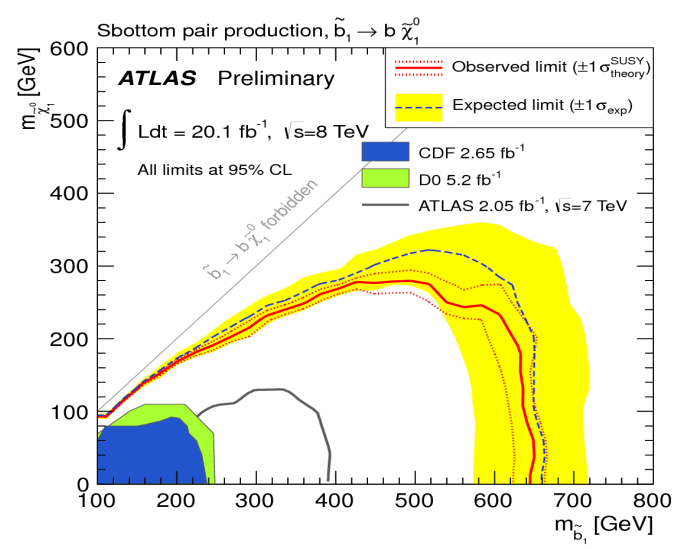

(b) Expected and observed exclusion limits at 95\% CL in $\left(m_{\tilde{b}}, m_{\tilde{\chi}_{1}^{0}}\right)$ plane.

Figure 1: Results of the search for sbottom pair production, assuming $\tilde{b} \rightarrow b \tilde{\chi}_{1}^{0}$. [3] 


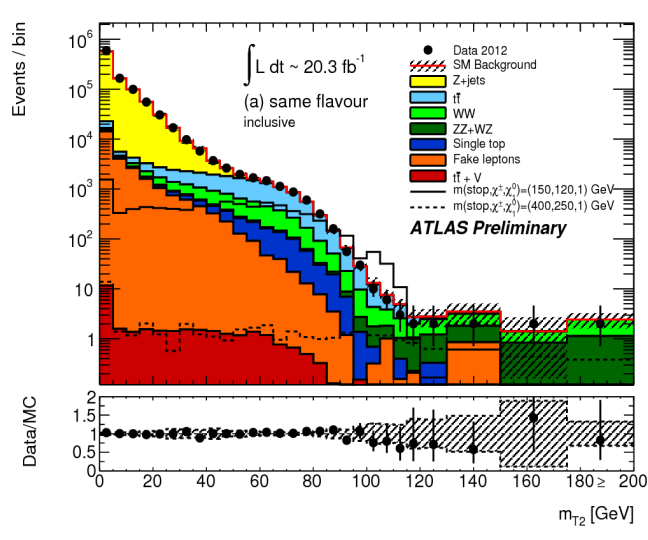

(a) Distribution of $m_{T 2}$ for 2 leptons same flavour SR targeting $\tilde{t} \rightarrow b \tilde{\chi}_{1}^{ \pm}[8]$.

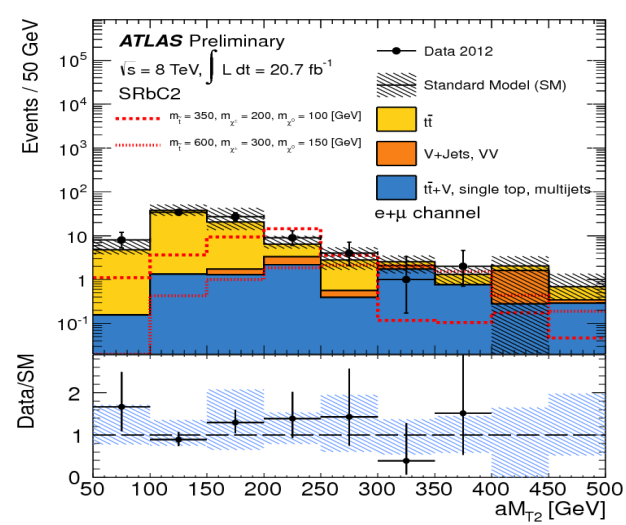

(b) Distribution of $a m_{T 2}$ for loose 1 lepton SR targeting $\tilde{t} \rightarrow b \tilde{\chi}_{1}^{ \pm}[6]$.

Figure 2: Significative distributions of 1 and 2 lepton SR for $\tilde{t} \rightarrow b \tilde{\chi}_{1}^{ \pm}$searches. On each plot, all requirements are applied except that of the shown distribution. The bands represent the total uncertainty.

event.

$$
\mathbf{p}_{b}^{\ell \ell}=\mathbf{p}_{T}^{\text {miss }}+\mathbf{p}_{T}^{\ell 1}+\mathbf{p}_{T}^{\ell_{2}}
$$

Different signal regions are defined for same and opposite lepton flavours and different tightness of the requirements applied. One of the final signal region of this analysis is shown in Figure 2(a).

The 1 lepton analysis strategy is based on a $2 b$-jets selection that exploits hard cuts on the $b$-jets momenta and the effective mass of the event (Eq. 4.3) to enhance the signal over background ratio.

$$
\mathrm{m}_{\mathrm{eff}}=\sum_{j e t} p_{T}^{j e t}+p_{T}^{\ell}+E_{\mathrm{T}}^{\mathrm{miss}}
$$

In addition, an asymmetric generalisation of the stransverse mass ([9],[10]) is exploited, that is explicitely designed to suppress di-leptonic $t \bar{t}$ background. In this case, it is assumed that one of the leptons from the $W$ decay is missed and contributes to the $E_{\mathrm{T}}^{\text {miss }}$ of the event. The same minimisation as in Eq.(4.1) is performed, this time $a, b$ being the system between the lepton and one $b$-jet and the other $b$-jet in the event, respectively. One of the missing particles is assumed to have the $W$ mass, so that the endpoint of the distribution is in this case the top mass.

A representative distribution $\left(a m_{T 2}\right)$ for the loosest of the 1 lepton signal regions is shown in Figure 2(b). The summary of all exclusion limits for $\tilde{t} \rightarrow b \tilde{\chi}_{1}^{ \pm}$decay channel are shown in the left frame of Fig. 3.

\section{Conclusions and perspectives}

A large amount of the SUSY natural phase space have been investigated and excluded from many ATLAS analyses, most of them based on the full $20128 \mathrm{TeV}$ dataset. They have contributed to narrow down a fraction of the parameter space of 3rd generation squarks direct production. However, challenging signatures might still hide in these $21 \mathrm{fb}^{-1}$ of data and future improvements 


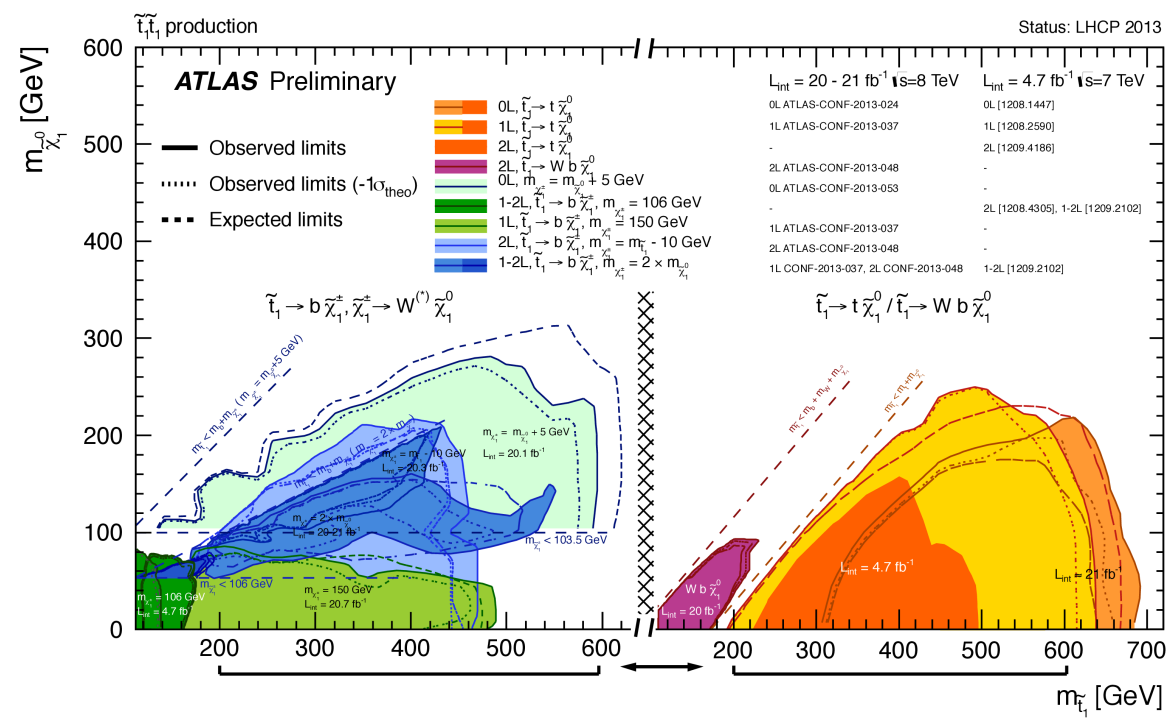

Figure 3: Summary of the dedicated ATLAS searches for top squark (stop) pair production. Exclusion limits at $95 \% \mathrm{CL}$ are shown in the $\left(\tilde{t}_{1}-\tilde{\chi}_{1}^{0}\right)$ mass plane. Three decay modes are considered separately with $100 \% \mathrm{BR}: \tilde{t}_{1} \rightarrow t+\tilde{\chi}_{1}^{0}, \tilde{t}_{1} \rightarrow W+b+\tilde{\chi}_{1}^{0}$ and $\tilde{t}_{1} \rightarrow b+\tilde{\chi}_{1}^{ \pm}$. [11]

are planned in order to fully exploit this dataset. Otherwise, the LHC run at design $\sqrt{s}$ will allow access to unexplored scenarios.

\section{References}

[1] ATLAS Collaboration, The ATLAS Experiment at the CERN Large Hadron Collider, JINST 3 (2008) S08003.

[2] Search for supersymmetry using events with three leptons, multiple jets, and missing transverse momentum in $13.0 \mathrm{fb}^{-1}$ of pp collisions with the ATLAS detector at $\sqrt{\mathrm{s}}=8 \mathrm{TeV}$, Tech. Rep. ATLAS-CONF-2012-151, CERN, Geneva, Nov, 2012.

[3] Search for direct third generation squark pair production in final states with missing transverse momentum and two b-jets in sqrts $=8 \mathrm{TeV}$ p p collisions with the ATLAS detector., Tech. Rep. ATLAS-CONF-2013-053, CERN, Geneva, May, 2013.

[4] D. Tovey, On measuring the masses of pair-produced semi-invisibly decaying particles at hadron colliders, JHEP 0804 (2008) 034, arXiv:0802 .2879 [hep-ph ] .

[5] Search for direct production of the top squark in the all-hadronic ttbar + etmiss final state in $21 \mathrm{fb}-1$ of p-pcollisions at $\operatorname{sqrt}(s)=8 \mathrm{TeV}$ with the ATLAS detector, Tech. Rep. ATLAS-CONF-2013-024, CERN, Geneva, Mar, 2013.

[6] Search for direct top squark pair production in final states with one isolated lepton, jets, and missing transverse momentum in sqrts $=8, T e V$ pp collisions using $21 \mathrm{fb}^{-1}$ of ATLAS data, Tech. Rep. ATLAS-CONF-2013-037, CERN, Geneva, Mar, 2013.

[7] ATLAS Collaboration, Search for direct stop production in events with missing transverse momentum and two b-jets using $12.8 \mathrm{fb}^{-1}$ of pp collisions at $\sqrt{s}=8 \mathrm{TeV}$ with the ATLAS detector, ATLAS-CONF-2013-001, Dec, 2012. 
[8] Search for direct top squark pair production in final states with two leptons in $\sqrt{s}=8 \mathrm{TeV} p p$ collisions using 20 $\mathrm{fb}^{-1}$ of ATLAS data., Tech. Rep. ATLAS-CONF-2013-048, CERN, Geneva, May, 2013.

[9] C. Lester and D. Summers, Measuring masses of semiinvisibly decaying particles pair produced at hadron colliders, Phys.Lett. B463 (1999) 99-103, arXiv : hep-ph/9906349 [hep-ph] .

[10] Y. Bai, H.-C. Cheng, J. Gallicchio, and J. Gu, Stop the Top Background of the Stop Search, JHEP 1207 (2012) 110, arXiv:1203.4813 [hep-ph].

[11] ATLAS Collaboration. Combined Summary Plots Public Twiki Page. 